\title{
Effects of MRP8, LPS, and Lenalidomide on the Expressions of TNF- $\alpha$, Brain-Enriched, and Inflammation-Related MicroRNAs in the Primary Astrocyte Culture
}

\author{
Ahmed Omran, ${ }^{1,2}$ Muhammad Usman Ashhab, ${ }^{1}$ Na Gan, ${ }^{1}$ Huimin Kong, \\ Jing Peng, ${ }^{1}$ and Fei Yin ${ }^{1}$ \\ ${ }^{1}$ Department of Pediatrics, Xiangya Hospital of Central South University, Changsha, Hunan 410008, China \\ ${ }^{2}$ Departments of Pediatrics and Neonatology, Suez Canal University, Ismailia 41522, Egypt
}

Correspondence should be addressed to Fei Yin; yf_2323@yahoo.com

Received 8 July 2013; Accepted 22 August 2013

Academic Editors: H. Budka, T. Matsuda, and T. Pannicke

Copyright (c) 2013 Ahmed Omran et al. This is an open access article distributed under the Creative Commons Attribution License, which permits unrestricted use, distribution, and reproduction in any medium, provided the original work is properly cited.

Astrocytes are now recognized as a heterogeneous class of cells with many important and diverse functions in healthy and diseased central nervous system (CNS). MicroRNAs (miRNAs) are small, noncoding RNAs which may have key roles in astrocytes activation in response to various stimuli. We performed quantitative real-time PCR (qPCR) to detect changes in the expressions of brain-enriched miRNAs $(124,134,9,132$, and 138), inflammation-related miRNAs (146a, 21, 181a, 221, and 222), and tumor necrosis factor alpha (TNF- $\alpha$ ) in the rat primary astrocyte cultures after stimulation with myeloid-related protein 8 (MRP8) and lipopolysaccharides (LPS). Further, we inhibited the expression of TNF- $\alpha$ in the astrocytes by using TNF- $\alpha$ inhibitor (lenalidomide) and tested for the first time the effect of this inhibition on the expressions of the same tested miRNAs. Stimulation of the astrocytes with MRP8 or LPS leads to significant upregulation of miRNAs $(124,134,9,132,146 a, 21,181$, 221, and 222), while miRNA-138 was downregulated. TNF- $\alpha$ inhibition with lenalidomide leads to opposite expressions of the tested miRNAs. These miRNAs may play an important role in activation of the astrocytes and may be a novel target for cell-specific therapeutic interventions in multiple CNS diseases.

\section{Introduction}

Astrocytes are dynamic cells considered as the most abundant cells in the central nervous system (CNS). In the last 3 decades it has become clear that astrocytes are responsible for a wide variety of essential and complex functions in the healthy CNS [1]. Astrocyte dysfunction is becoming a recognized feature in multiple brain diseases including migraine, epilepsy, leukodystrophies, inflammatory demyelinating diseases, infections, brain edema and metabolic disorders, metal intoxications, neurodegenerative disorders, and schizophrenia $[2,3]$.

In response to inflammatory or other pathological conditions, astrocytes leave their quiescent state and become activated in a process known as astrogliosis [4]. This is associated with initiation and regulation of CNS immune response via the release of proinflammatory cytokines tumor necrosis factor alpha (TNF- $\alpha$ ), interleukin $1-\beta$ (IL-1 $\beta)$, and interleukin-6 (IL-6) [5].

MicroRNAs (miRNAs) are small, nonprotein-coding RNA molecules that modulate gene translation. Their expressions are altered in multiple CNS, cardiovascular, and chronic diseases [6-8]. Sixty to seventy percent of the known miRNAs are expressed in the brain, while some of those are enriched or unique to neuronal tissues $[9,10]$. MiRNAs are also recognized as vital regulators in immunity and inflammation making some of the identified miRNAs be inflammationrelated [11, 12]. Expressional levels of miRNAs changed when the astrocytes are subjected to different stimuli such as lipopolysaccharide (LPS) and interferon gamma (IFN- $\gamma$ ) [13] or oxygen-glucose deprivation (OGD) [14].

Activated astrocyte is a major source of TNF- $\alpha$ in the inflamed CNS, which plays an important role in the pathogenesis of multiple CNS diseases such as brain trauma, 
ischemic injury, multiple sclerosis, and Alzheimer's disease [15].

Lenalidomide is a derivative of thalidomide that belongs to a class of drugs known as immunomodulating drugs [16]. With respect to the parent drug, thalidomide, lenalidomide is a more potent inhibitor of TNF- $\alpha$ and has a weaker antiangiogenic effect.

In this study, we demonstrate the changes in the expressional levels of brain-enriched miRNAs $(124,134,9,132$, and 138) and inflammation-related miRNAs (146a, 21, 181a, 221, and 222), when the astrocytes are subjected to stimulation with proinflammatory mediators (myeloid-related protein 8 (MRP8) and LPS). Further, we examined for the first time the effect of lenalidomide (TNF- $\alpha$ inhibitor) followed by stimulation with MRP8 and LPS on the expressions of the same miRNAs.

\section{Materials and Methods}

2.1. Primary Astrocyte Culture. Astrocytes were obtained from cerebral cortices of male neonatal Sprague-Dawley (SD) rats. The animals were decapitated and the brains were fetched immediately and placed in phosphate-buffered saline (PBS) at $4^{\circ} \mathrm{C}$. After removing the meninges and blood vessels, the tissues were minced, washed, centrifuged, and incubated in $0.025 \%(\mathrm{w} / \mathrm{v})$ trypsin and $60 \mathrm{mg} / \mathrm{mL}$ DNase for $15-20$ minutes. High-glucose Dulbecco's Modified Eagle Medium (DMEM) (Highclone, USA) containing 10\% (v/v) fetal bovine serum (FBS) is added to complete the trypsinization. Cells were cultured in DMEM supplemented with $10 \% \mathrm{FBS}, 2 \mathrm{mM}$ L-glutamine, 100 units $/ \mathrm{mL}$ penicillin, and $100 \mu \mathrm{g} / \mathrm{mL}$ streptomycin and were maintained at $37^{\circ} \mathrm{C}$ under $>90 \%$ humidity and $5 \% \mathrm{CO}_{2}$. The culture medium was renewed twice a week till cells reached confluence then the cells were purified by repeated trypsinization and inoculated at a density of $2.5 \times 10^{4}$ cells $/ \mathrm{cm}^{2}$ at least 4 times. More than $95 \%$ of the cultured cells were astrocytes as identified by immunofluorescent staining for glial fibrillary acidic protein (GFAP). Experiments were carried out 21 days after culturing of the astrocytes.

\subsection{Stimulation of the Astrocytes with MRP8 and LPS. Part of} the prepared astrocytes was divided into three groups.

Group A. Astrocytes are cultured without serum DMEM medium for 30 hours to detect the expressions of the tested brain-enriched miRNAs, inflammation-related miRNA, and TNF- $\alpha$ in the resting astrocytes.

Group B. Astrocytes are cultured without serum DMEM medium for 6 hours then with DMEM and MRP8 $(0.5 \mu \mathrm{g} / \mathrm{mL})$ for 24 hours [17], to detect the effect of astrocytes stimulation with MRP8 on the expressions of the tested brain-enriched miRNAs, inflammation-related miRNAs, and TNF- $\alpha$.

Group C. Astrocytes are cultured without serum DMEM medium for 6 hours then with DMEM and LPS ((1000 ng/mL), SIGMA, USA) for 24 hours [18], to detect the effect of astrocytes stimulation with LPS on the expressions of the tested brain-enriched miRNAs, inflammation-related miRNAs, and TNF- $\alpha$.

2.3. Stimulation of the Astrocytes with MRP8 and LPS after TNF- $\alpha$ Inhibition with Lenalidomide. Other part of the prepared astrocytes was divided into two other groups.

Group D. Astrocytes are cultured without serum DMEM medium containing TNF- $\alpha$ inhibitor lenalidomide $((2 \mu \mathrm{M})$, Selleckchem, USA) for 6 hours and then cultured with DMEM medium with MRP8 for 24 hours to detect the effect of TNF- $\alpha$ inhibition on the tested brain-enriched and inflammation-related miRNAs expression using TNF- $\alpha$ inhibitor followed by astrocyte stimulation with MRP8.

Group E. Astrocytes are cultured without serum DMEM medium containing TNF- $\alpha$ inhibitor lenalidomide $((2 \mu \mathrm{M})$, Selleckchem, USA) for 6 hours and then cultured with DMEM medium with LPS for 24 hours to detect the effect of TNF- $\alpha$ inhibition on the tested brain-enriched and inflammation-related miRNAs expression using TNF- $\alpha$ inhibitor followed by astrocyte stimulation with LPS.

2.4. RNA Isolation from Astrocytes. For RNA isolation, the astrocytes were washed with $0.01 \mathrm{mM}$ PBS to clean the medium first. Then $1 \mathrm{~mL}$ of Trizol per $1 \times 10^{6}$ astrocytes was added and collected in $1.5 \mathrm{~mL}$ Eppendorf tubes. After addition of $0.2 \mathrm{~mL}$ chloroform, the aqueous phase was isolated using phase-lock tubes (Eppendorf, Hamburg, Germany). RNA was precipitated with $0.5 \mathrm{~mL}$ isopropyl alcohol, washed twice with $75 \%$ ethanol, and dissolved in nuclease-free water. The concentration and purity of RNA were determined at 260/280 nm using a nanodrop spectrophotometer (Ocean Optics, Dunedin, FL, USA).

\subsection{Expressions of the Tested Brain-Enriched and Inflamma-} tion-Related miRNAs in the Different Astrocytes Groups by Quantitative PCR ( $q P C R)$. cDNA synthesis was performed from the RNA extracted from the different prepared astrocyte groups using the One Step PrimeScript miR cDNA Synthesis Kit (TAKARA, Dalian, China) which includes three mixes (2X miRNA Reaction Buffer Mix, miR PrimeScript RT Enzyme Mix, and $0.1 \%$ BSA). A $10 \mu \mathrm{L}$ reaction contained $5 \mu \mathrm{L} 2 \mathrm{X}$ miR reaction buffer mix, $1 \mu \mathrm{L}$ miR PrimeScript RT enzyme mix, and $1 \mu \mathrm{L} 0.1 \%$ BSA. RNA 100 pg, and DEPCtreated water up to $10 \mu \mathrm{L}$. The tubes were incubated at $37^{\circ} \mathrm{C}$ for $60 \mathrm{~min}$; the reaction was terminated at $85^{\circ} \mathrm{C}$ for $5 \mathrm{sec}$, and then the reaction was held at $4^{\circ} \mathrm{C}$. qPCR was performed using the SYBRR Premix Ex Taq II (TAKARA, Dalian, China) kit in triplicate. The $10 \mu \mathrm{L}$ PCR contained the following: $5 \mu \mathrm{L}$ SYBRR Premix Ex Taq II, $0.4 \mu \mathrm{L}$ Uni-miR qPCR Primer, $2 \mu \mathrm{L}$ miR specific primer for the tested miRNA (GeneCopoeia, USA), $1 \mu \mathrm{L} \mathrm{cDNA}$, and 1.6 $\mu \mathrm{L}$ DEPC-treated water. The PCRs were incubated at $50^{\circ} \mathrm{C}$ for $2 \mathrm{~min}$ (UDG incubation) and $95^{\circ} \mathrm{C}$ for $30 \mathrm{sec}$, followed by 40 cycles of $95^{\circ} \mathrm{C}$ for $5 \mathrm{sec}$ and $60^{\circ} \mathrm{C}$ for $30 \mathrm{sec}$, followed by melting curve analysis from 65.0 to $95.0^{\circ} \mathrm{C}$ (increment $0.5^{\circ} \mathrm{C}, 0: 05$ ). The relative expression 
TABLE 1: Means and SD of the brain-enriched and inflammation-related miRNAs in astrocytes after stimulation with (MRP8 and LPS) and inhibition of TNF- $\alpha$ with lenalidomide.

\begin{tabular}{lccccc}
\hline MiRNAs & Resting astrocytes & MRP8 stimulated & LPS stimulated & MRP8 + lenalidomide & LPS + lenalidomide \\
\hline MiRNA-124 & $0.490 \pm 0.036$ & $1.083 \pm 0.076$ & $0.983 \pm 0.028$ & $0.200 \pm 0.050$ & $0.283 \pm 0.028$ \\
MiRNA-134 & $0.433 \pm 0.028$ & $1.167 \pm 0.057$ & $1.100 \pm 0.100$ & $0.116 \pm 0.028$ & $0.116 \pm 0.028$ \\
MiRNA-9 & $0.333 \pm 0.028$ & $1.033 \pm 0.057$ & $0.966 \pm 0.057$ & $0.166 \pm 0.028$ & $0.133 \pm 0.028$ \\
MiRNA-132 & $0.450 \pm 0.050$ & $1.100 \pm 0.100$ & $1.033 \pm 0.057$ & $0.233 \pm 0.028$ & $0.233 \pm 0.028$ \\
MiRNA-138 & $0.383 \pm 0.028$ & $0.166 \pm 0.028$ & $0.150 \pm 0.050$ & $0.800 \pm 0.200$ & $0.700 \pm 0.100$ \\
MiRNA-146a & $0.416 \pm 0.028$ & $0.900 \pm 0.100$ & $0.966 \pm 0.057$ & $0.100 \pm 0.0$ & $0.116 \pm 0.028$ \\
MiRNA-21 & $0.300 \pm 0.050$ & $0.900 \pm 0.100$ & $0.916 \pm 0.076$ & $0.133 \pm 0.028$ & $0.116 \pm 0.028$ \\
MiRNA-181a & $0.350 \pm 0.050$ & $0.966 \pm 0.057$ & $0.916 \pm 0.076$ & $0.176 \pm 0.025$ & $0.166 \pm 0.028$ \\
MiRNA-221 & $0.266 \pm 0.028$ & $0.966 \pm 0.057$ & $0.900 \pm 0.100$ & $0.100 \pm 0.0$ & $0.116 \pm 0.028$ \\
MiRNA-222 & $0.316 \pm 0.028$ & $0.966 \pm 0.057$ & $0.933 \pm 0.057$ & $0.150 \pm 0.050$ & $0.183 \pm 0.028$ \\
\hline
\end{tabular}

levels for brain-enriched miRNAs $(124,134,9,132$, and 138) and inflammations-related miRNAs (146a, 21, 181a, 221, and 221) were calculated using the comparative CT method. The expression of the U6 small nuclear RNA gene was used as an internal control.

2.6. TNF- $\alpha$ Expression by Quantitative PCR (qPCR) in the Different Astrocyte Groups. cDNA was generated from the different astrocyte groups using PrimeScript RT Reagent Kit (TAKARA, Dalian, China) which includes four reagents: $5 \mathrm{X}$ PrimeScriptTM Buffer (for real time), PrimeScriptTM RT Enzyme Mix I, Oligo dT Primer, and Random 6 mers. A $10 \mu \mathrm{L}$ reaction contained $2 \mu \mathrm{L} 5 \mathrm{X}$ PrimeScriptTM Buffer, $0.5 \mu \mathrm{L}$ PrimeScriptTM RT Enzyme Mix I, $0.5 \mu$ L Oligo dT Primer, and $0.5 \mu \mathrm{L}$ Random 6 mers. RNA $100 \mathrm{pg}-1 \mathrm{ng}$ and RNase-free $\mathrm{dH}_{2} \mathrm{O}$ water up to $10 \mu \mathrm{L}$. The tubes were incubated at $37^{\circ} \mathrm{C}$ for $15 \mathrm{~min}$; the reactions were terminated at $85^{\circ} \mathrm{C}$ for $5 \mathrm{sec}$, and then the reactions were held at $4^{\circ} \mathrm{C}$. TNF- $\alpha$ expressions were analyzed using SYBR Premix Ex Taq II (TAKARA). qPCR was performed in triplicate. The $10 \mu \mathrm{L}$ PCR contained the following: $5 \mu \mathrm{L}$ SYBRR Premix ExTaq II, $1 \mu \mathrm{L}$ Specific Primer TNF- $\alpha$-F (Sangon, China), $1 \mu$ L Specific Primer TNF$\alpha$-R (Sangon, China), $1 \mu \mathrm{L} \mathrm{cDNA}$, and $2 \mu \mathrm{L}$ DEPC-treated water. The PCRs were incubated at $50^{\circ} \mathrm{C}$ for $2 \mathrm{~min}$ (UDG incubation) and $95^{\circ} \mathrm{C}$ for $30 \mathrm{sec}$, followed by 40 cycles of $95^{\circ} \mathrm{C}$ for $5 \mathrm{sec}$ and $60^{\circ} \mathrm{C}$ for $30 \mathrm{sec}$, followed by melting curve analysis from 65.0 to $95.0^{\circ} \mathrm{C}$ (increment $0.5^{\circ} \mathrm{C}, 0: 05$ ). The relative expression levels for TNF- $\alpha$ were calculated using the comparative CT method. $\beta$-Actin was used as an internal control.

2.7. Statistical Analysis. All of the data are expressed as means \pm standard deviation (SD). A Student's $t$-test was performed to determine significant differences between two groups. One-way analysis of variance (ANOVA) was utilized to determine significant differences among multiple groups. $P<0.05$ was considered to be statistically significant.

\section{Results}

3.1. Relative Expressions of Brain-Enriched and InflammationRelated miRNAs in Astrocytes after Stimulation with MRP8 or LPS. qPCR results showed significant upregulation of miRNAs $(124,134,9,132,146 \mathrm{a}, 21,181 \mathrm{a}, 221$, and 222) in the astrocytes after stimulation with MRP8 or LPS compared to resting astrocytes, while miRNA-138 was significantly downregulated (means and SD values are given in Table 1). miRNAs expressions were normalized to that of the rat U6B small nuclear RNA gene (rnu6b) (Figures 1(a), 1(b), 1(c), 1(d), $1(\mathrm{e}), 1(\mathrm{f}), 1(\mathrm{~g}), 1(\mathrm{~h}), 1(\mathrm{i})$, and $1(\mathrm{j}))$.

3.2. TNF- $\alpha$ Relative Expression in the Astrocytes after Stimulation with MRP8 or LPS and Inhibition with Lenalidomide. qPCR results showed significant upregulation of TNF- $\alpha$ in the astrocytes after stimulation with MRP8 or LPS compared to resting (resting mean $0.300 \pm 0.0$, MRP8 mean $0.850 \pm$ 0.050 , and LPS mean $0.750 \pm 0.050, P<0.05$ ). TNF- $\alpha$ expression showed significant downregulation after inhibition with TNF- $\alpha$ inhibitor (lenalidomide) compared to stimulated astrocytes (lenalidomide + MRP8 mean $0.166 \pm 0.028$; lenalidomide + LPS mean $0.116 \pm 0.028, P<0.05)$. TNF- $\alpha$ expression was normalized to that of $\beta$-actin (Figure 2).

3.3. Relative Expressions of Brain-Enriched and InflammationRelated miRNAs in the Astrocytes after TNF- $\alpha$ Inhibition with Lenalidomide Followed by Stimulation with MRP8 and LPS. qPCR results showed significant downregulation of miRNAs $(124,134,9,132,146 a, 21,181 a, 221$, and 222) in the astrocytes after TNF- $\alpha$ inhibition with lenalidomide compared to their expressions in the resting astrocytes while miRNA138 showed the opposite pattern of expression (means and $\mathrm{SD}$ values are given in Table 1). MiRNAs expressions were normalized to that of the rat U6B small nuclear RNA gene (rnu6b) (Figures 3(a), 3(b), 3(c), 3(d), 3(e), 3(f), 3(g), 3(h), $3(\mathrm{i})$, and $3(\mathrm{j}))$.

\section{Discussion}

Astrocytes, constituting the major glial cell population in the CNS, are playing a crucial role in many developmental and physiological functions $[19,20]$. Marked alterations in astrocyte function are a universal response to CNS disease or injury. Reactive astrocytes may provide neuroprotective 


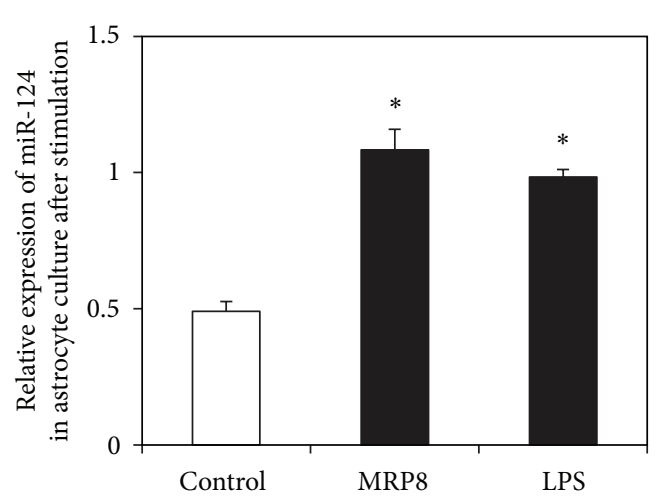

(a)

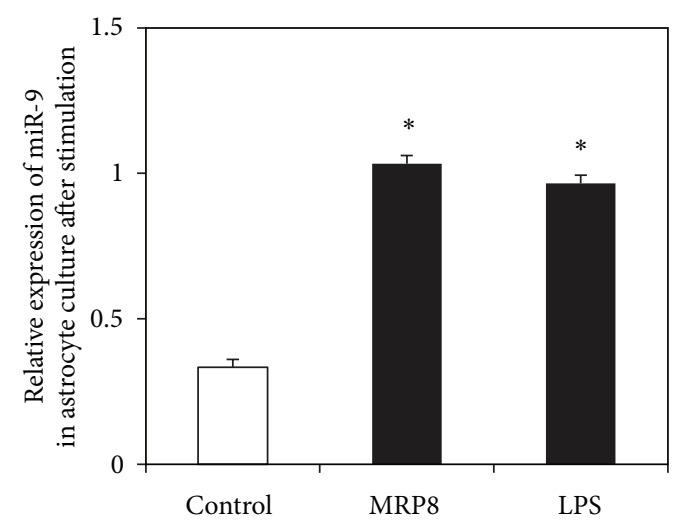

(c)

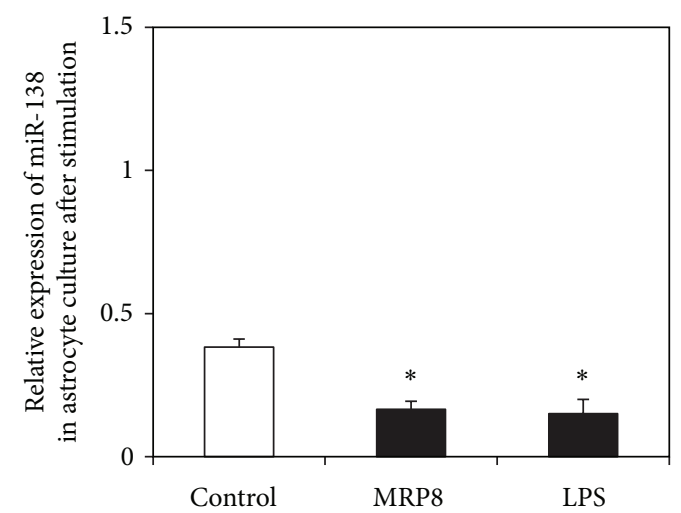

(e)

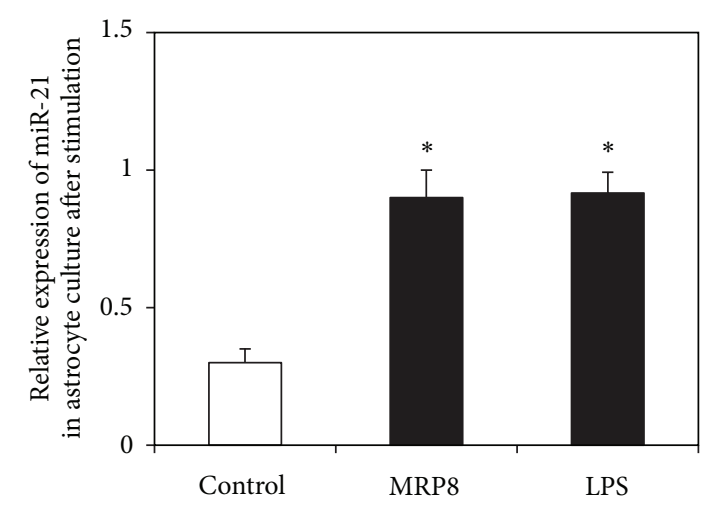

(g)

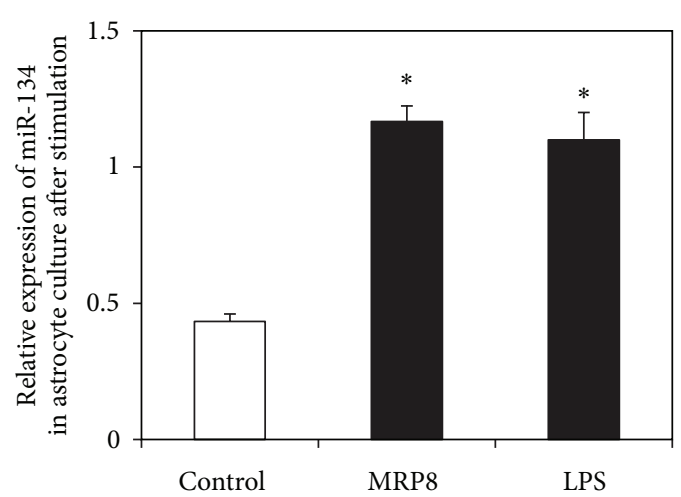

(b)

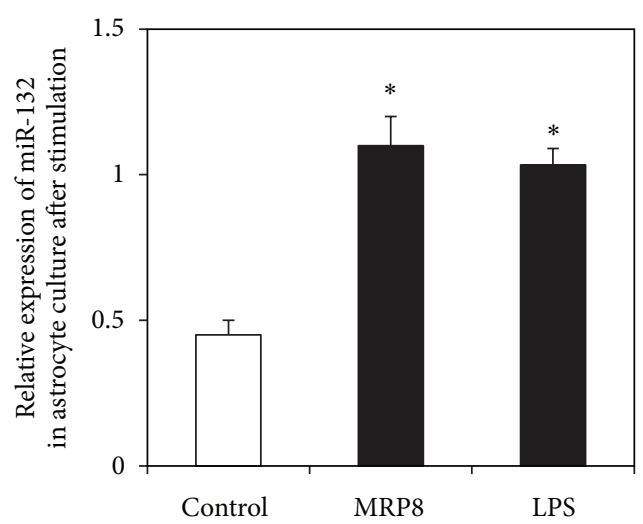

(d)

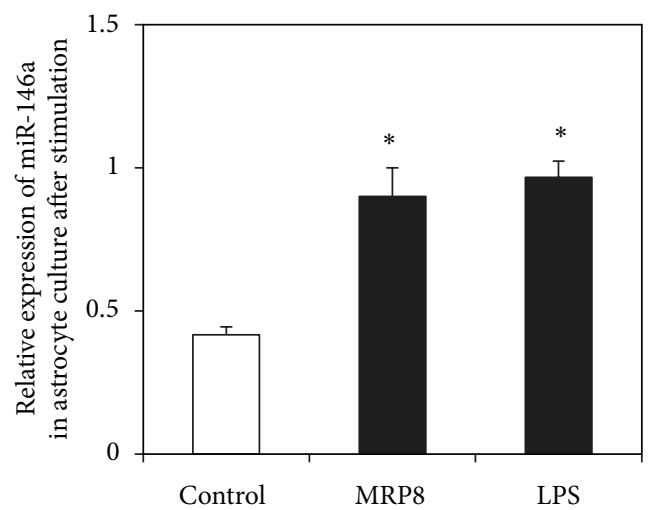

(f)

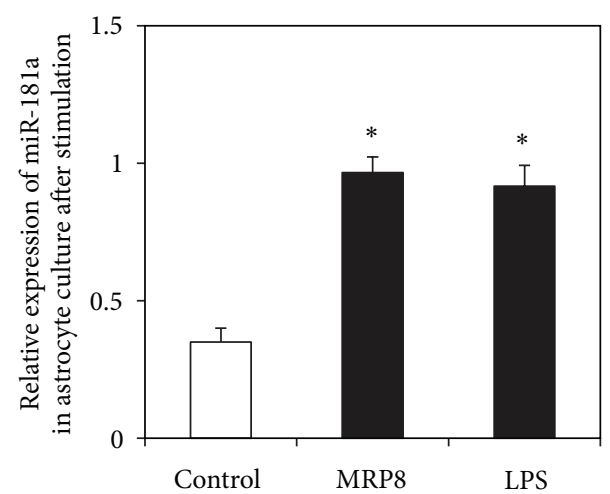

(h)

FIGURe 1: Continued. 


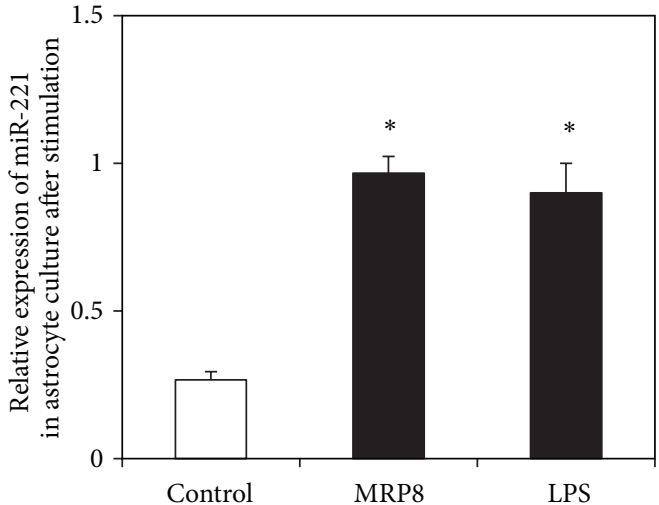

(i)

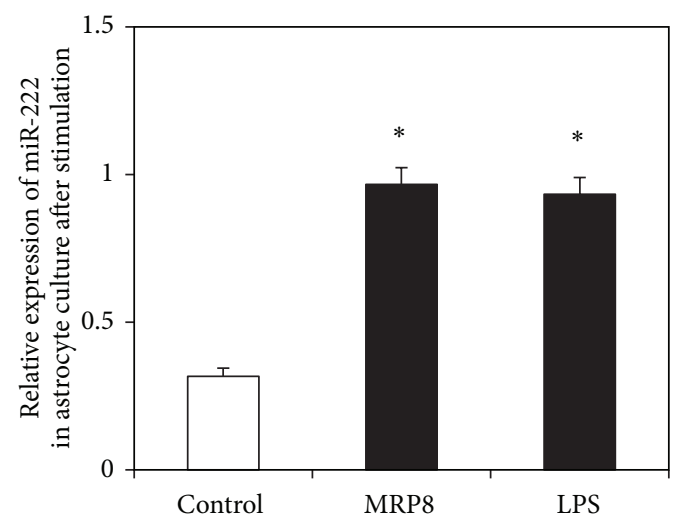

(j)

Figure 1: ((a), (b), (c), (d), (f), (g), (h), (i), (j)) qPCR results showed significant upregulation of miRNAs (124, 134, 9, 132, 146a, 21, 181a, 221, and 222) in the astrocytes after stimulation with MRP8 and LPS, while miRNA-138 was significantly downregulated in the astrocytes after stimulation with MRP8 and LPS (e).

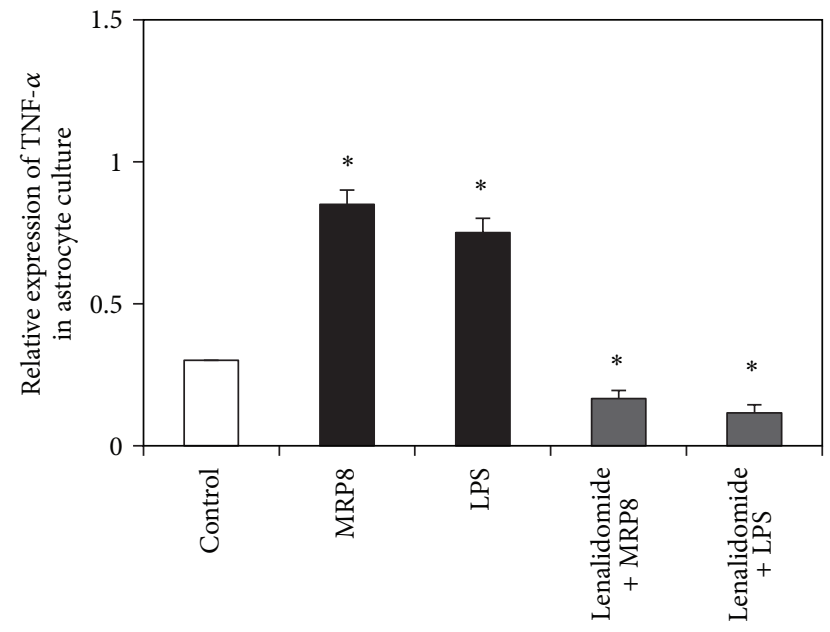

FIGURE 2: qPCR results showed significant upregulation of TNF$\alpha$ in the astrocytes after stimulation with LPS and MRP8 and significant downregulation after inhibition with lenalidomide (TNF- $\alpha$ inhibitor) compared to stimulated astrocytes.

effects in the early stage of the injury whereas at a later stage the formation of glial scar inhibits CNS regeneration [21].

The discovery of miRNAs functions reveals novel posttranscriptional regulation that controls or fine-tunes the transcriptional output. An increasing body of the literature supports the critical role of miRNAs in several biological processes of the CNS, as well as in the pathogenesis of different disorders including epilepsy, neurodegenerative, vascular, and neuroinflammatory disorders [22-26].

MiRNAs have critical roles in neuronal and astroglial development and cell fate decision. Deletion of miRNAprocessing enzyme Dicer in neural progenitor cells was found to control the switch of neurogenesis to gliogenesis [27, 28]. Selective deletion of Dicer in the cerebellar astrocytes leads to global disruption of the astroglial miRNA biogenesis [29]. Moreover, miRNA-125b and miRNA-146a are involved in astroglial cell proliferation and in the innate immune and inflammatory response [30].

A better understanding of molecular mechanisms underlying astrocytes activation in response to stimulation with MRP8 and LPS, through detecting the changes in brainenriched and inflammation-related miRNAs, may provide new step in understanding the pathogenesis of multiple brain disorders. In the first part of this experiment, we focused on the expressional changes in the brain-enriched miRNAs (124, 134, 9, 132, and 138) and inflammation-related miRNAs (146a, $21,181 a, 221$, and 222) in the primary astrocyte culture in vitro, 24 hours after stimulation with MRP8 and LPS.

We found that brain-enriched miRNAs $(124,134,9$, and 132) showed significant upregulation in the astrocytes 24 hours after stimulation with MRP8 and LPS, while miRNA138 takes the opposite pattern of expression. Mor et al. [13] have demonstrated a global shift in miRNA expression in astrocytes treated with LPS and IFN- $\gamma$. Although miRNA124 is the most abundant brain-specific miRNA, there is a limited data about the importance of this miRNA in astrocyte function. Just very recently, Morel et al. [31] suggested that miRNA-124 can broadly modulate astroglial gene expression. MiRNA-124 has a very important role in maintaining the microglia in the quiescent state [32].

MiRNA-134 is brain-specific, implicated in the control of neuronal microstructure [33]. MiRNA-134 silencing exerts prolonged seizure suppressant and neuroprotective actions [34]. The exact function of miRNA-134 upregulation in the stimulated astrocytes needs further research.

Initially identified as brain-specific, miRNA- 9 is a highly functional miRNA in brain development, which has been involved in the fine-tuning of nuclear factor Kappa-B- (NF$\kappa \mathrm{B}-$ ) dependent inflammatory response [35]. As an NF- $\kappa \mathrm{B}-$ mediated miRNA which plays a central role in CNS relevant stress and neuropathology, this may explain its upregulation in our experiment.

MiRNA-132 was recently designated as a "NeurimmiR," a class of miRs suggested to act as a crosstalk between the neuronal and immune system [36]. MiRNA-132 was 


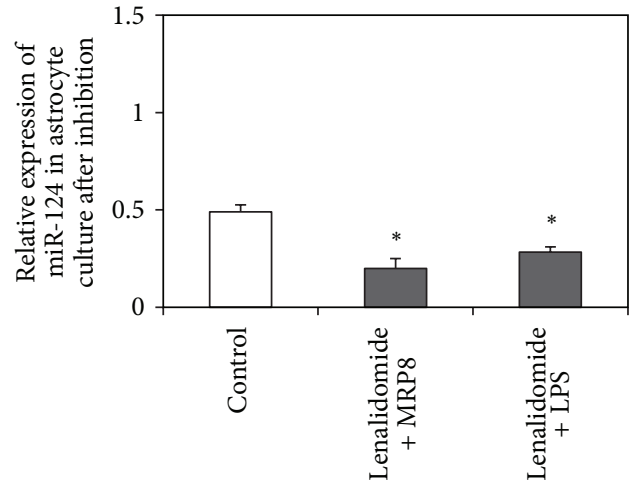

(a)

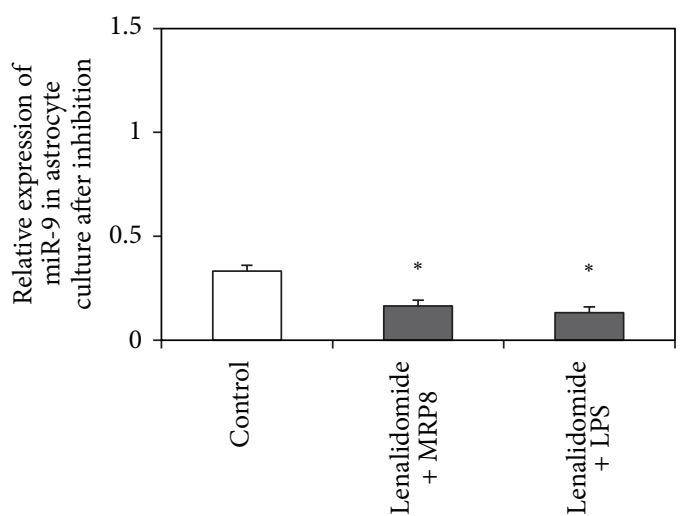

(c)

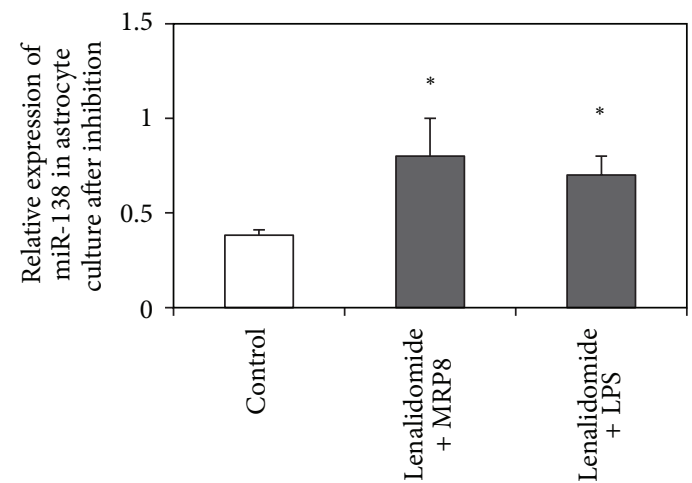

(e)

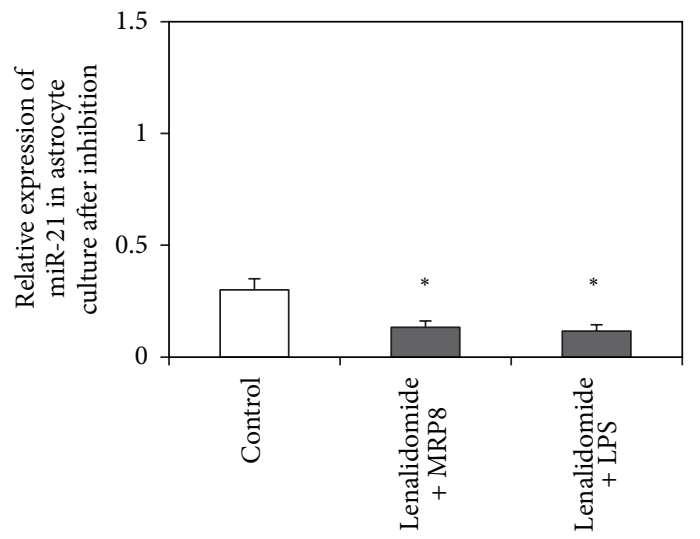

(g)

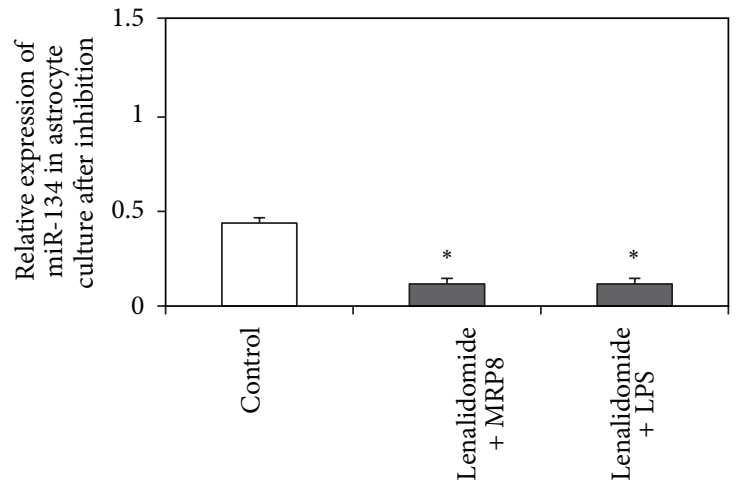

(b)

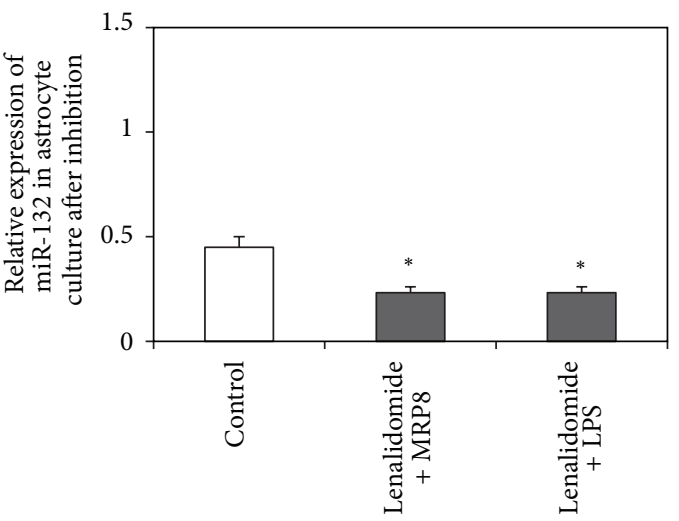

(d)

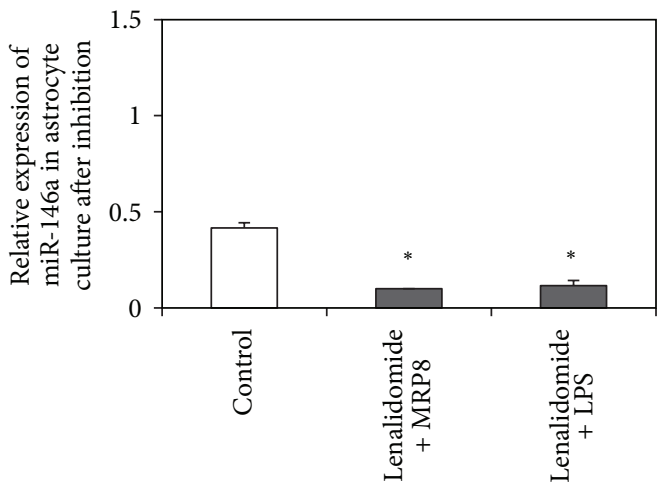

(f)

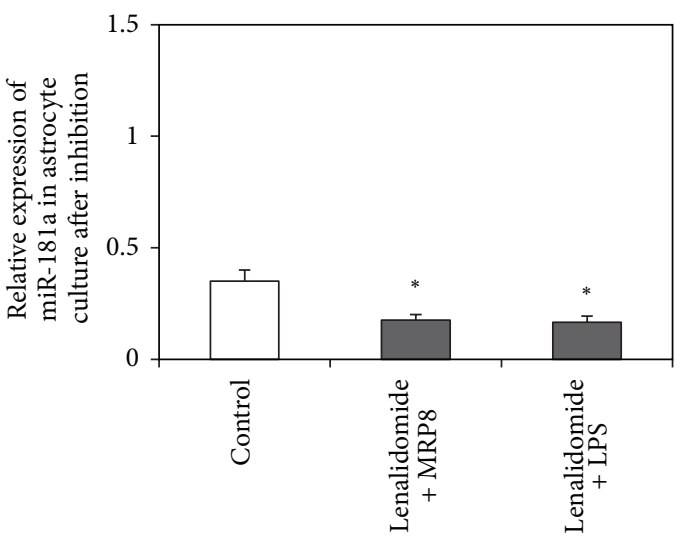

(h)

Figure 3: Continued. 


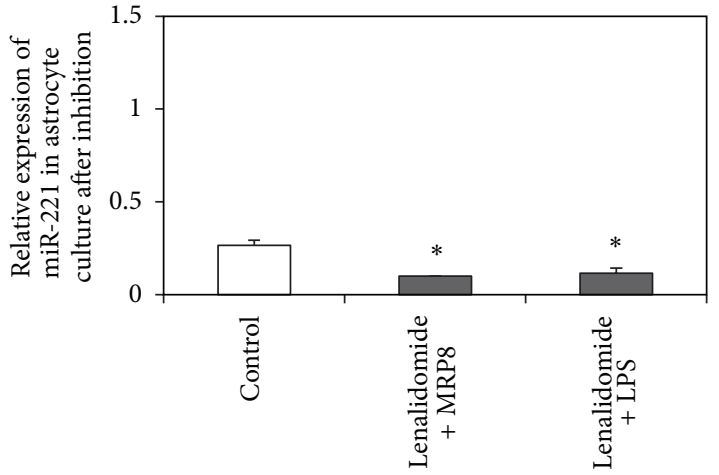

(i)

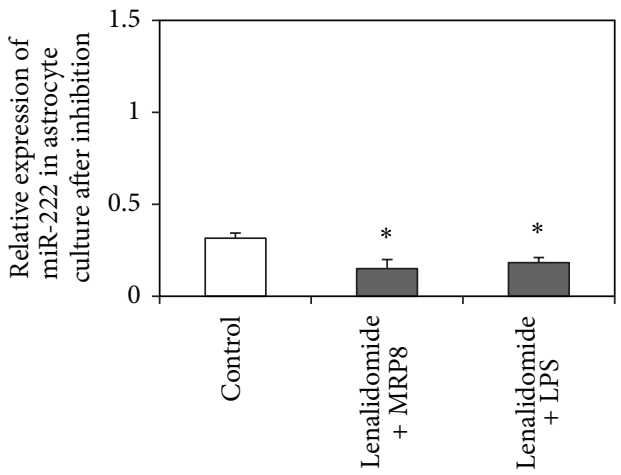

(j)

Figure 3: ((a), (b), (c), (d), (f), (g), (h), (i), (j)) qPCR results showed significant downregulation of miRNAs (124, 134, 9, 132, 146a, 21, 181a, 221, and 222) after inhibition with lenalidomide compared to stimulated astrocytes, while miRNA-138 was significantly upregulated after inhibition with lenalidomide compared to stimulated astrocytes (e).

upregulated in the activated astrocyte in our experiment; it was also upregulated in animal model of mesial temporal lobe epilepsy characterized by significant astrocyte activation [23].

In our experiment miRNA-138 was the only brainenriched miRNA that was significantly downregulated in the stimulated astrocytes. MiRNA-138 was also downregulated in animal model after status epilepticus [37]. The exact role for this miRNA in astrocyte and the mechanism of its downregulation in response stimulation with MRP8 and LPS need further experiments.

Reactive astrocytes are playing essential roles in regulating CNS inflammation [21]. In response to different kinds of stimulation, reactive astrocytes can make many different kinds of molecules with either pro- or anti-inflammatory potential $[21,38]$. Increasing evidence supports the involvement of miRNAs in the regulation of inflammation in human neurological disorders and various miRNAs are also considered to represent a new class of mediators of inflammation $[11,39]$.

We found that inflammation-related miRNAs (146a, 21, 181a, 221, and 222) showed significant upregulation in the astrocytes 24 hours after stimulation with MRP- 8 and LPS.

miRNA-146a was upregulated in the activated astrocyte in our work. Mor et al. [13] reported in their study activation of miRNA-146a in the astrocyte activation process in response to LPS and INF- $\gamma$. Upregulation of miRNA-146a has been also detected in active multiple sclerosis lesions [40], in human Alzheimer's disease brain [41] and in TLE [22, 42], suggesting a key role of this miRNA in governing astrocyte activation and function in these pathologies. Iyer et al. [43] found that miRNA-146a was induced as a negative-feedback regulator of the astrocyte-mediated inflammatory response and suggested a role for miRNA-146a-mediated regulation of inflammation in glial cells.

Due to the critical function of miRNA-21 in various inflammatory signaling pathways, miRNA-21 has become an attractive and promising target in inflammation-related diseases. We observed that the expression of miRNA-21 was upregulated in the stimulated astrocytes. Ziu et al. [14] found upregulation of miRNA-21 in the astrocytes exposed to OGD. Also miRNA-21 has a novel role in regulating astrocytic hypertrophy and glial scar progression after spinal cord injury [44].

MiRNA-181a which plays a critical role in the inflammatory response and development of immune system [45] was found to be also upregulated in the activated astrocytes. Reduction of miRNA-181a levels is associated with reduced cell death, reduced oxidative stress, and preserved mitochondrial function in astrocytes [46].

MiRNA-221 and miRNA-222 are two highly homologous miRNAs, whose dysregulations have been recently described in several types of human tumors. MiRNA-221 and miRNA222 were upregulated in the activated astrocytes. MiRNA-221 was upregulated in the human astrocytic tumors [47]. Astrocytes activated with LPS and INF- $\gamma$ showed upregulation of miR-222 [13].

TNF- $\alpha$ is a proinflammatory cytokine that not only acts upon astrocytes but is also produced by them in response to specific signals. Available data would suggest that TNF$\alpha$ is involved in the induction of a reactive astrogliosis and may also play a role in CNS repair process. In our study we found significant upregulation of TNF- $\alpha$ expression when the astrocytes are stimulated with MRP8 or LPS while astrocytes treated with lenalidomide followed by stimulation with MRP8 or LPS showed significant downregulation of TNF- $\alpha$ expression.

In the second part of the present study, we examined for the first time the effect of lenalidomide (TNF- $\alpha$ inhibitor) on the expressions of the tested brain-enriched and inflammation-related miRNAs in the astrocytes.

We found that treating the astrocytes with lenalidomide for 6 hours, followed by stimulation with MRP8 or LPS for 24 hours, is associated with significant downregulation in miRNAs $(124,134,9,132,146 a, 21,181 a, 221$, and 222) which are previously upregulated when the astrocytes were stimulated with MRP8 or LPS, while miRNA-138 surprisingly showed the opposite pattern of expression to be significantly upregulated in response to treatment with lenalidomide. This 
novel finding may indirectly link the changes that occur in the tested miRNAs in response to stimulation with MRP8 and LPS to the changes that occur in the TNF- $\alpha$ expression, which is reversed with treating the astrocytes with TNF- $\alpha$ inhibitor.

In conclusion, our study shows that exposure of astrocytes to MRP8 and LPS leads to significant changes in the expressions of brain-enriched and inflammation-related miRNAs and TNF- $\alpha$. These changes are reversed when the astrocytes are treated with TNF- $\alpha$ inhibitor. These miRNAs may play a role in activation of the astrocytes and may be a novel target for cell-specific therapeutic interventions in multiple CNS diseases.

\section{Conflict of Interests}

None of the authors have any conflict of interests to disclose.

\section{Authors' Contribution}

Ahmed Omran and Muhammad Usman Ashhab equally shared in this study.

\section{Acknowledgments}

This work was kindly supported by the National Natural Science Foundation of China (nos. 81201371, 81171226, and 81100846) and Doctoral Innovation Project, Hunan, China (CX2012B087).

\section{References}

[1] M. V. Sofroniew and H. V. Vinters, "Astrocytes: biology and pathology," Acta Neuropathologica, vol. 119, no. 1, pp. 7-35, 2010.

[2] J. De Keyser, J. P. Mostert, and M. W. Koch, "Dysfunctional astrocytes as key players in the pathogenesis of central nervous system disorders," Journal of the Neurological Sciences, vol. 267, no. 1-2, pp. 3-16, 2008.

[3] G. Seifert, K. Schilling, and C. Steinhäuser, "Astrocyte dysfunction in neurological disorders: a molecular perspective," Nature Reviews Neuroscience, vol. 7, no. 3, pp. 194-206, 2006.

[4] P. A. Carpentier, W. S. Begolka, J. K. Olson, A. Elhofy, W. J. Karpus, and S. D. Miller, "Differential activation of astrocytes by innate and adaptive immune stimuli," Glia, vol. 49, no. 3, pp. 360-374, 2005.

[5] Y. Dong and E. N. Benveniste, "Immune function of astrocytes," Glia, vol. 36, no. 2, pp. 180-190, 2001.

[6] A. Omran, D. Elimam, S. Shalaby, J. Peng, and F. Yin, "MicroRNAs: a light into the "Black Box" of neuropediatric diseases?" Neuromolecular Medicine, vol. 14, no. 4, pp. 244-261, 2012.

[7] A. Omran, D. Elimam, K. Webster, L. Shehadeh, and F. Yin, "MicroRNAs: a new piece in the paediatric cardiovascular disease puzzle," Cardiology in the Young, vol. 23, no. 5, pp. 642$655,2013$.

[8] A. Omran, D. Elimam, and F. Yin, "MicroRNAs: new insights into chronic childhood diseases," BioMed Research International, vol. 2013, Article ID 291826, 13 pages, 2013.

[9] M. Bak, A. Silahtaroglu, M. Møller et al., "MicroRNA expression in the adult mouse central nervous system," RNA, vol. 14, no. 3, pp. 432-444, 2008.
[10] S. K. Fineberg, K. S. Kosik, and B. L. Davidson, "MicroRNAs potentiate neural development," Neuron, vol. 64, no. 3, pp. 303309, 2009.

[11] E. Sonkoly and A. Pivarcsi, "MicroRNAs in inflammation," International Reviews of Immunology, vol. 28, no. 6, pp. 535-561, 2009.

[12] R. M. O'Connell, D. S. Rao, A. A. Chaudhuri, and D. Baltimore, "Physiological and pathological roles for microRNAs in the immune system," Nature Reviews Immunology, vol. 10, no. 2, pp. 111-122, 2010.

[13] E. Mor, Y. Cabilly, Y. Goldshmit et al., "Species-specific microRNA roles elucidated following astrocyte activation," Nucleic Acids Research, vol. 39, no. 9, pp. 3710-3723, 2011.

[14] M. Ziu, L. Fletcher, S. Rana, D. F. Jimenez, and M. Digicaylioglu, "Temporal differences in microRNA expression patterns in astrocytes and neurons after ischemic injury," PLOS ONE, vol. 6, no. 2, Article ID e14724, 2011.

[15] K. Akassoglou, E. Douni, J. Bauer, H. Lassmann, G. Kollias, and L. Probert, "Exclusive tumor necrosis factor (TNF) signaling by the p75TNF receptor triggers inflammatory ischemia in the CNS of transgenic mice," Proceedings of the National Academy of Sciences of the United States of America, vol. 100, no. 2, pp. 709-714, 2003.

[16] J. Blake Marriott, G. Muller, D. Stirling, and A. G. Dalgleish, "Immunotherapeutic and antitumour potential of thalidomide analogues," Expert Opinion on Biological Therapy, vol. 1, no. 4, pp. 675-682, 2001.

[17] T. Vogl, K. Tenbrock, S. Ludwig et al., "Mrp8 and Mrp14 are endogenous activators of Toll-like receptor 4, promoting lethal, endotoxin-induced shock," Nature Medicine, vol. 13, no. 9, pp. 1042-1049, 2007.

[18] B. M. Kucher and J. T. Neary, "Bi-functional effects of ATP/P2 receptor activation on tumor necrosis factor-alpha release in lipopolysaccharide-stimulated astrocytes," Journal of Neurochemistry, vol. 92, no. 3, pp. 525-535, 2005.

[19] M. R. Freeman, "Specification and morphogenesis of astrocytes," Science, vol. 330, no. 6005, pp. 774-778, 2010.

[20] B. A. Barres, "The mystery and magic of Glia: a perspective on their roles in health and disease," Neuron, vol. 60, no. 3, pp. 430440, 2008.

[21] M. V. Sofroniew, "Molecular dissection of reactive astrogliosis and Glial scar formation," Trends in Neurosciences, vol. 32, no. 12, pp. 638-647, 2009.

[22] A. Omran, J. Peng, C. Zhang et al., "Interleukin-1 $\beta$ and microRNA-146a in an immature rat model and children with mesial temporal lobe epilepsy," Epilepsia, vol. 53, no. 7, pp. 12151224, 2012.

[23] J. Peng, A. Omran, M. U. Ashhab et al., "Expression patterns of miR-124, miR-134, miR-132, and miR-21 in an immature rat model and children with mesial temporal lobe epilepsy," Journal of Molecular Neuroscience, vol. 50, no. 2, pp. 291-297, 2013.

[24] M. U. Ashhab, A. Omran, H. Kong et al., "Expressions of tumor necrosis factor-alpha and microRNA-155 in immature rat model of status epilepticus and children with mesial temporal lobe epilepsy," Journal of Molecular Neuroscience, 2013.

[25] P. Sethi and W. J. Lukiw, "Micro-RNA abundance and stability in human brain: specific alterations in Alzheimer's disease temporal lobe neocortex," Neuroscience Letters, vol. 459, no. 2, pp. 100-104, 2009.

[26] C. Urbich, A. Kuehbacher, and S. Dimmeler, "Role of microRNAs in vascular diseases, inflammation, and angiogenesis," Cardiovascular Research, vol. 79, no. 4, pp. 581-588, 2008. 
[27] Y. Kawase-Koga, G. Otaegi, and T. Sun, "Different timings of dicer deletion affect neurogenesis and gliogenesis in the developing mouse central nervous system," Developmental Dynamics, vol. 238, no. 11, pp. 2800-2812, 2009.

[28] K. Zheng, H. Li, Y. Zhu, Q. Zhu, and M. Qiu, "MicroRNAs are essential for the developmental switch from neurogenesis to gliogenesis in the developing spinal cord," Journal of Neuroscience, vol. 30, no. 24, pp. 8245-8250, 2010.

[29] J. Tao, H. Wu, Q. Lin et al., "Deletion of astroGlial dicer causes non-cell autonomous neuronal dysfunction and degeneration," Journal of Neuroscience, vol. 31, no. 22, pp. 8306-8319, 2011.

[30] A. I. Pogue, M. E. Percy, J.-G. Cui et al., "Up-regulation of NF$\mathrm{kB}$-sensitive miRNA-125b and miRNA-146a in metal sulfatestressed human astroGlial (HAG) primary cell cultures," Journal of Inorganic Biochemistry, vol. 105, no. 11, pp. 1434-1437, 2011.

[31] L. Morel, M. Regan, H. Higashimori et al., "Neuronal exosomal miRNA dependent translational regulation of astroGlial glutamate transporter GLT1," The Journal of Biological Chemistry, vol. 288, no. 10, pp. 7105-7116, 2013.

[32] E. D. Ponomarev, T. Veremeyko, N. Barteneva, A. M. Krichevsky, and H. L. Weiner, "MicroRNA-124 promotes microGlia quiescence and suppresses EAE by deactivating macrophages via the C/EBP- $\alpha$-PU.1 pathway," Nature Medicine, vol. 17, no. 1, pp. 64-70, 2011.

[33] G. M. Schratt, F. Tuebing, E. A. Nigh et al., "A brain-specific microRNA regulates dendritic spine development," Nature, vol. 439, no. 7074, pp. 283-289, 2006.

[34] E. M. Jimenez-Mateos, T. Engel, P. Merino-Serrais et al., "Silencing microRNA-134 produces neuroprotective and prolonged seizure-suppressive effects," Nature Medicine, vol. 18, no. 7, pp. 1087-1094, 2012.

[35] F. Bazzoni, M. Rossato, M. Fabbri et al., "Induction and regulatory function of miR-9 in human monocytes and neutrophils exposed to proinflammatory signals," Proceedings of the National Academy of Sciences of the United States of America, vol. 106, no. 13, pp. 5282-5287, 2009.

[36] H. Soreq and Y. Wolf, "NeurimmiRs: microRNAs in the neuroimmune interface," Trends in Molecular Medicine, vol. 17, no. 10, pp. 548-555, 2011.

[37] R. M. Risbud and B. E. Porter, "Changes in MicroRNA expression in the whole hippocampus and Hippocampal Synaptoneurosome Fraction following Pilocarpine Induced Status Epilepticus," PLoS ONE, vol. 8, no. 1, Article ID e53464, 2013.

[38] G. R. John, S. C. Lee, and C. F. Brosnan, "Cytokines: powerful regulators of Glial cell activation," Neuroscientist, vol. 9, no. 1, pp. 10-22, 2003.

[39] S. R. Quinn and L. A. O’Neill, "A trio of microRNAs that control Toll-like receptor signalling," International Immunology, vol. 23, no. 7, pp. 421-425, 2011.

[40] A. Junker, M. Krumbholz, S. Eisele et al., "MicroRNA profiling of multiple sclerosis lesions identifies modulators of the regulatory protein CD47," Brain, vol. 132, no. 12, pp. 3342-3352, 2009.

[41] J. G. Cui, Y. Y. Li, Y. Zhao, S. Bhattacharjee, and W. J. Lukiw, "Differential regulation of Interleukin-1 Receptor-associated Kinase-1 (IRAK-1) and IRAK-2 by microRNA-146a and NF- $\kappa$ B in stressed human astroGlial cells and in Alzheimer disease," Journal of Biological Chemistry, vol. 285, no. 50, pp. 3895138960, 2010.

[42] E. Aronica, K. Fluiter, A. Iyer et al., "Expression pattern of miR146a, an inflammation-associated microRNA, in experimental and human temporal lobe epilepsy," European Journal of Neuroscience, vol. 31, no. 6, pp. 1100-1107, 2010.
[43] A. Iyer, E. Zurolo, A. Prabowo et al., "MicroRNA-146a: a key regulator of astrocyte-mediated inflammatory response," PLoS ONE, vol. 7, no. 9, Article ID e44789, 2012.

[44] O. G. Bhalala, L. Pan, V. Sahni et al., "microRNA-21 regulates astrocytic response following spinal cord injury," The Journal of Neuroscience, vol. 32, no. 50, pp. 17935-17947, 2012.

[45] W. Xie, Z. Li, M. Li, N. Xu, and Y. Zhang, "miR-181a and inflammation: miRNA homeostasis response to inflammatory stimuli in vivo," Biochemical and Biophysical Research Communications, vol. 430, no. 2, pp. 647-652, 2013.

[46] Y.-B. Ouyang, Y. Lu, S. Yue, and R. G. Giffard, "MiR-181 targets multiple Bcl-2 family members and influences apoptosis and mitochondrial function in astrocytes," Mitochondrion, vol. 12, no. 2, pp. 213-219, 2012.

[47] A. Conti, M. Aguennouz, D. La Torre et al., "miR-21 and 221 upregulation and miR-181b downregulation in human grade IIIV astrocytic tumors," Journal of Neuro-Oncology, vol. 93, no. 3, pp. 325-332, 2009. 

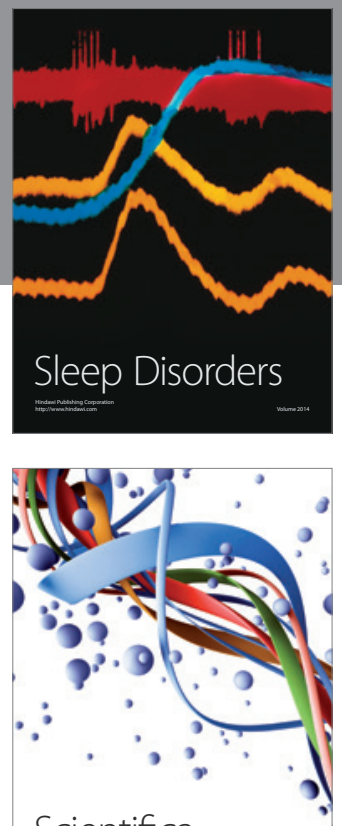

Scientifica
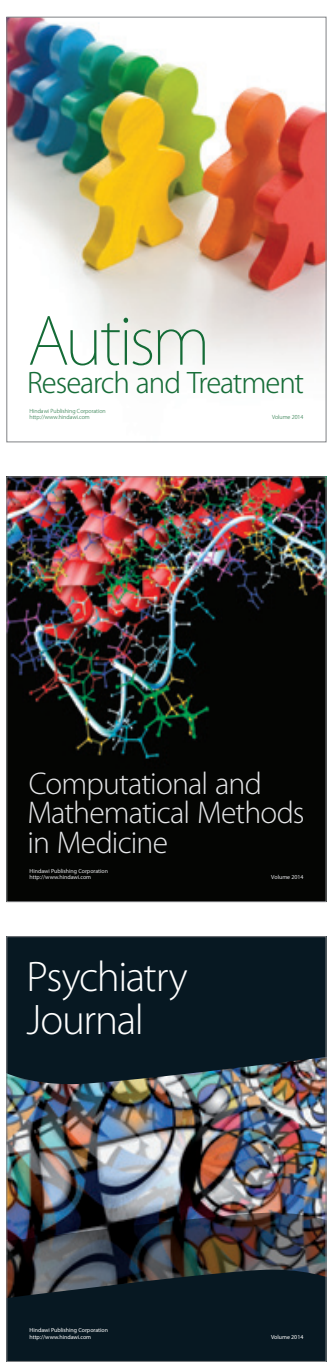
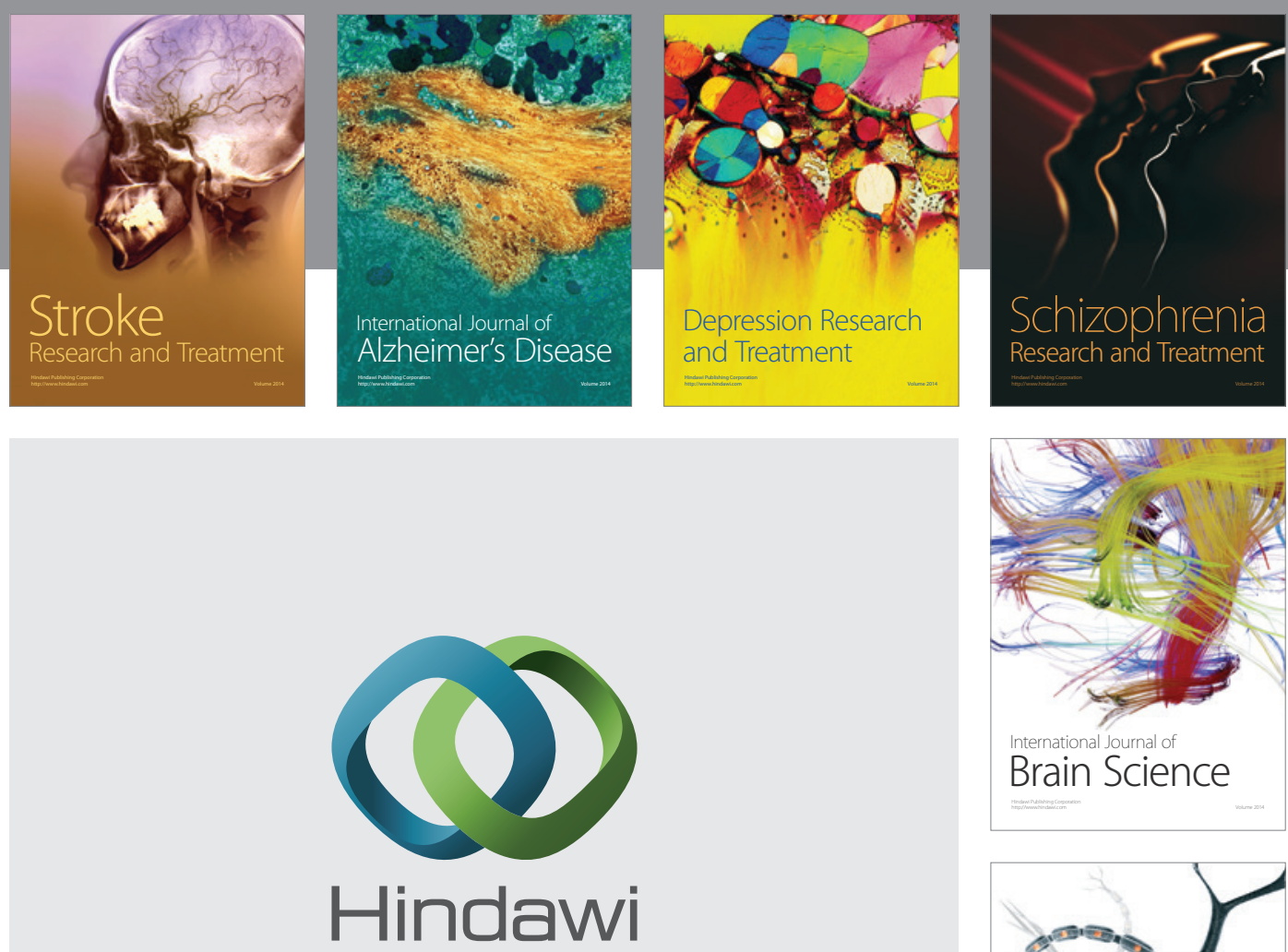

Submit your manuscripts at

http://www.hindawi.com
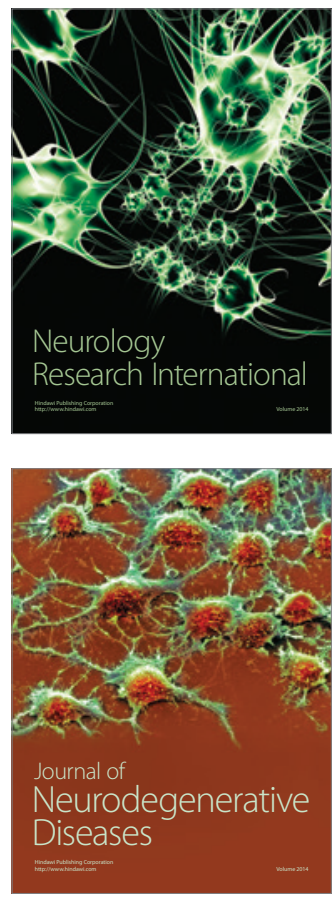

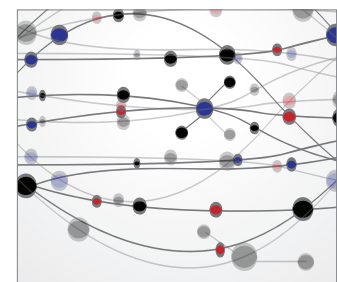

The Scientific World Journal
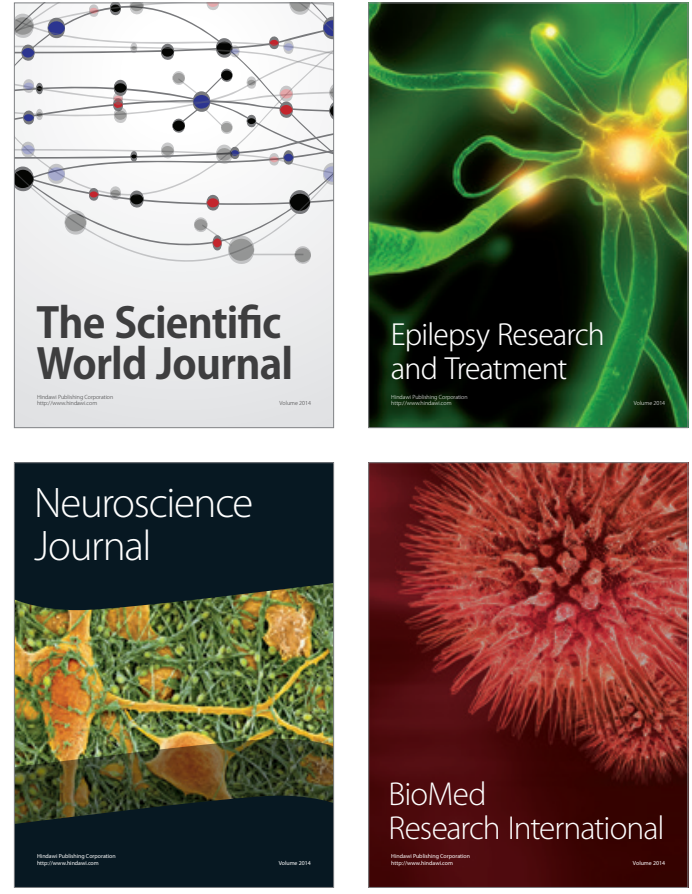

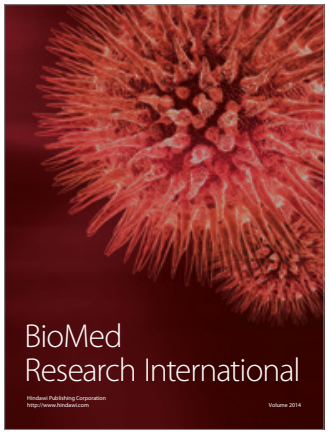

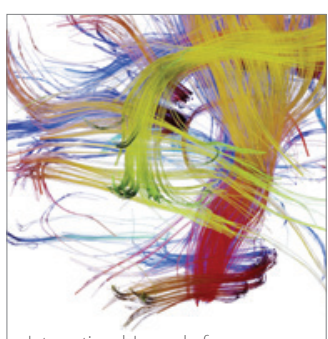

Brain Science

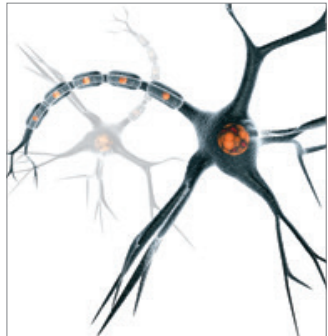

Neural Plasticity
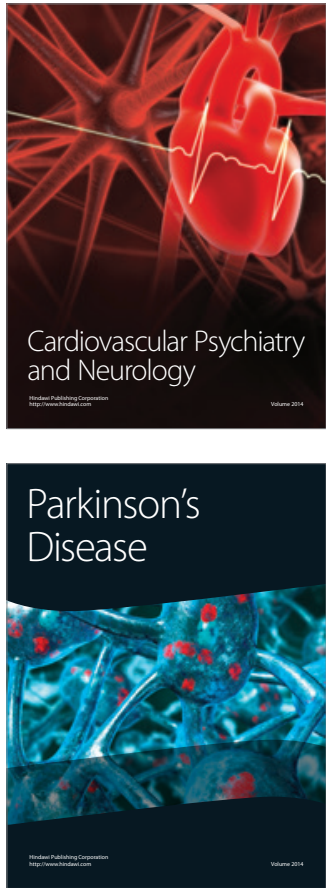